\title{
Multistatic acoustic characterization of seabed targets
}

Erin M. Fischell, and Henrik Schmidt

Citation: The Journal of the Acoustical Society of America 142, 1587 (2017);

View online: https://doi.org/10.1121/1.5002887

View Table of Contents: http://asa.scitation.org/toc/jas/142/3

Published by the Acoustical Society of America

\section{Articles you may be interested in}

Source localization in an ocean waveguide using supervised machine learning

The Journal of the Acoustical Society of America 142, 1176 (2017); 10.1121/1.5000165

Spherical wave scattering from rough surfaces and array processing: Application to sound-speed profile measurement uncertainty analysis

The Journal of the Acoustical Society of America 142, 1189 (2017); 10.1121/1.5000234

Deep-water measurements of container ship radiated noise signatures and directionality

The Journal of the Acoustical Society of America 142, 1563 (2017); 10.1121/1.5001063

Measured and modeled acoustic propagation underneath the rough Arctic sea-ice

The Journal of the Acoustical Society of America 142, 1619 (2017); 10.1121/1.5003786

Performance comparisons of frequency-difference and conventional beamforming

The Journal of the Acoustical Society of America 142, 1663 (2017); 10.1121/1.5003787

A metric for characterization of two-dimensional spatial coherence

The Journal of the Acoustical Society of America 142, EL313 (2017); 10.1121/1.5001163 


\title{
Multistatic acoustic characterization of seabed targets
}

\author{
Erin M. Fischell ${ }^{\mathrm{a})}$ and Henrik Schmidt \\ Department of Mechanical Engineering, Massachusetts Institute of Technology, Cambridge, \\ Massachusetts 02139, USA
}

(Received 24 April 2017; revised 11 August 2017; accepted 29 August 2017; published online 25 September 2017)

One application for autonomous underwater vehicles (AUVs) is detecting and classifying hazardous objects on the seabed. An acoustic approach to this problem has been studied in which an acoustic source insonifies seabed target while receiving AUVs with passive sensing payloads discriminate targets based on features of the three dimensional scattered fields. The OASES-SCATT simulator was used to study how scattering data collected by mobile receivers around targets insonified by mobile sources might be used for sphere and cylinder target characterization in terms of shape, composition, and size. The impact of target geometry on these multistatic scattering fields is explored, and a discrimination approach developed in which the source and receiver circle the target with the same radial speed. The frequency components of the multistatic scattering data at different bistatic angles are used to form models for target characteristics. Data are then classified using these models. Classification accuracies were greater than $98 \%$ for shape and composition. Regression for target volume showed potential, with $90 \%$ chance of errors less than $15 \%$. The significance of this approach is to make classification using low-cost vehicles plausible from scattering amplitudes and the relative angles between the target, source, and receiver vehicles.

(C) 2017 Acoustical Society of America. [http://dx.doi.org/10.1121/1.5002887]

[AMT]

Pages: $1587-1596$

\section{INTRODUCTION}

Autonomous underwater vehicles (AUVs) are used in a variety of defense, oceanographic and commercial applications. One AUV application of particular interest is the mission of autonomously localizing and classifying seabed targets. This is important in a security context in mine countermeasures (MCM), and also for the detection and remediation of unexploded ordinance (UXOs), as discussed across the literature including by Blondel, Zampolli, and others. ${ }^{1-3}$ Existing MCM sensing techniques (such as sidescan and synthetic aperture sonar) are expensive, power-intensive, and produce images difficult for a low-power computer to interpret autonomously. We have been studying an alternate acoustic approach in which an acoustic source insonifies seabed targets while AUVs discriminate targets based on features sensed in the resulting three dimensional (3D) scattered fields. The goal for this approach is to develop a system that can be deployed on multiple inexpensive, low-powered AUVs that can perform target localization and classification in real time without human intervention. This mission is of interest because of the new generation of lowcost AUVs such as the General Dynamics Bluefin SandShark (shown in Fig. 1) or Riptide micro-UUV (unmanned underwater vehicle) that might be used in multivehicle networks for detecting and classifying targets, but do not have the space or power for conventional MCM sensors.

These AUVs would be fitted with inexpensive hydrophone nose arrays and onboard signal processing payloads to calculate a target's scattered amplitude as a function of the

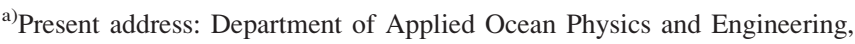
Woods Hole Oceanographic Institution, Woods Hole, MA 02543, USA. Electronic-mail: efischell@whoi.edu
}

angles between the source and receiver (bistatic angle) and the angle between the source and target major axis (aspect angle) (Fig. 2). Initial work in two 2014 bistatic scattering experiments successfully demonstrated data collection, signal processing, and simple target classification in the fixedsource, or bistatic, configuration. ${ }^{4,5}$ However, these experiments revealed an issue with the fixed-source geometry: the aspect angle (angle between the target's major axis and the acoustic source) significantly affects the target's radiation pattern. This paper describes our approach to solving this problem by looking instead at the multistatic scattered field that results when both acoustic source and receiver are mobile, resulting in the capture of multiple target aspect angles.

Multistatic scattering (source and receiver are mobile and separate) is well-studied in the radar context, ${ }^{6-8}$ and has

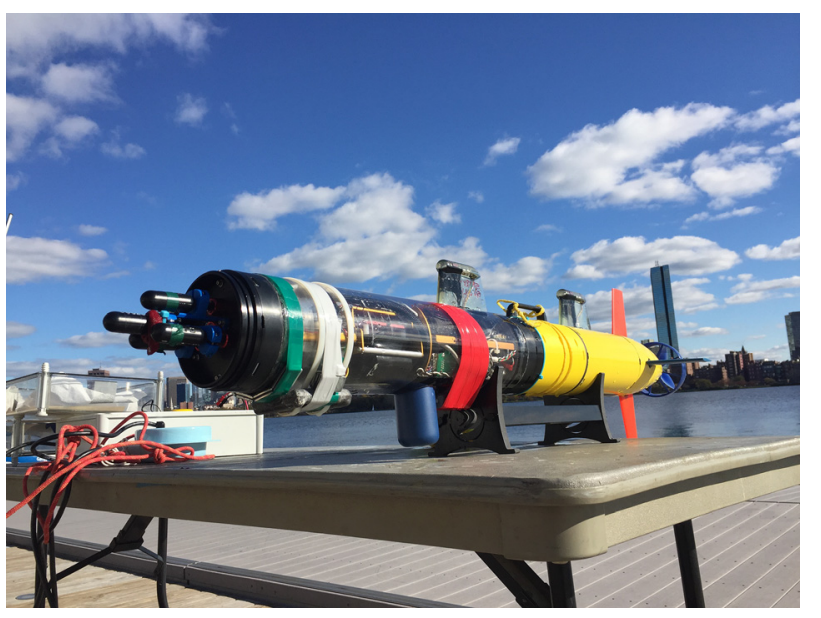

FIG. 1. (Color online) SandShark AUV with acoustics payload and hydrophone array. 


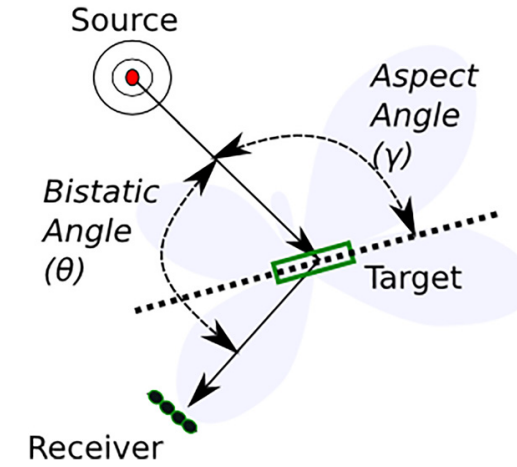

FIG. 2. (Color online) Aspect angle $(\gamma)$ and bistatic angle $(\theta)$ relative to source, receiver, and target as seen from above.

also been investigated in the past in the underwater context in simulation, tank, and field experiments. Simulation studies have been conducted in the multistatic space for spherical and cylindrical targets by Schmidt, Zampolli, and others to explore the implications of multiple mobile sources and receivers in a sonar network. ${ }^{9-11}$ Tank and pond experiments by Dobbins, Blondel, Waters, and others ${ }^{12-16}$ looked at different aspect angles on targets in a partial investigation of the multistatic scattering space. Ji et al. describe simulation and tank experiments that include data collection on cylinders to examine impact of aspect and bistatic angle on scattering over a limited range of bistatic angles. ${ }^{17}$ The GOATS'98 (Ref. 18) and SITAR experiments ${ }^{2}$ are field trials that have looked at in situ scattering from seabed targets. Multiaspect data were collected in both experiments for partial coverage of the multistatic space. Additional experiments that looked at bistatic scattering (fixed source) are well-summarized by Blondel and Pace. ${ }^{19}$ A more common approach is to look at the monostatic return from targets at different aspect angles for a sweep of frequencies (acoustic color): examples include work by Williams et al., ${ }^{20}$ Zampolli et al., ${ }^{21}$ and Bucaro et al. ${ }^{22}$

In terms of work on how to use multistatic acoustic data, Yildiz et al. ${ }^{23}$ have looked at how to process multistatic data from sonar networks to get localization and tracking using inversion. Lum et al. also looked at multistatic target localization. ${ }^{24}$ Synthetic imaging for classification in this context has also be explored, ${ }^{18}$ but is complicated by vehicle navigation uncertainty of not one but two or more AUVs. Tesei et al. looked at target parameter estimation of cylindrical shells based on target resonance response. ${ }^{25}$ In the most relevant prior work, classification of target in the multistatic space using the L4 norm versus scattering angle was investigated by Blondel in tank experiments. ${ }^{13}$ Blondel looked at scattering for three target aspect angles and multiple corresponding bistatic angles for targets with different composition, and found differences between the targets when multiple bistatic angles were observed. He also observed that the bistatic angle impacted the strength of the classification. While the feature space described by Blondel does not fully match what would be easily collected by multiple underwater vehicles, it shows that additional information is gleaned by varying both bistatic and aspect angle and suggested that that information may be used for classification by geometry and composition.

This paper looks at the specific case of how multistatic scattering data collected by mobile, AUV-based receivers around targets insonified by mobile, AUV-based sources might be used for target characterization. This initial investigation was conducted using the OASES-SCATT scattering simulator ${ }^{26}$ so that the full range of bistatic and aspect angles could be viewed. By looking at all possible bistatic angles $(0$ to $359 \mathrm{deg}$ ) and all possible aspect angles (0 to $180 \mathrm{deg}$ ), we had access to a richer potential feature space for target characterization than that used by Blondel. With the scattering simulator, we were able to investigate the impact of target composition, geometry, and environmental factors on multistatic scattering fields from spheres and cylinders, and to develop target discrimination approaches. First, multistatic scattering is described and some basic simulation results shown. AUV data collection behaviors and data analysis techniques for eliminating target orientation offsets are then presented. We then explain some target characterization techniques using the processed data. Results for target classification and parameter estimation using virtual-AUV-sampled acoustic data are then discussed, along with conclusions and suggestions for future work.

\section{MULTISTATIC SCATTERING METHODOLOGY}

When an object is insonified by an acoustic source, it reradiates that acoustic signal. Re-radiation consists of interfering time-delayed echoes, resulting in a $3 \mathrm{D}$ radiation pattern containing minima and maxima. The exact features of that $3 \mathrm{D}$ radiation pattern, or scattered field, are determined by factors such as frequency, target geometry, target composition, and environment. The radiation pattern changes for non-symmetric targets, such as cylinders, with aspect angle, which is determine by the acoustic source direction relative to the target's major axis. A multistatic scattering field is what is sensed by a receiver at different bistatic angles when the source aspect angle changes.

\section{A. Scattering field function}

The multistatic scattering field changes as a function of bistatic and aspect angles, as well as depth and range to the target. The dependence on bistatic and aspect angle is significantly stronger than the dependence on range or depth when looking at longer ranges: the general radiation pattern as a function of bistatic and aspect angle persists across range and depth because of the interference pattern caused by multipath in the waveguide. This effect is illustrated in Fig. 3: in the halfspace case, the target scattering continues to spread in a spherical pattern, with variations of up to $40 \mathrm{~dB}$ looking at simulated scattering field versus depth at a range of $20 \mathrm{~m}$. Looking at a range of $20 \mathrm{~m}$ in the waveguide case, the variations are smaller, only $15 \mathrm{~dB}$ or so. In the waveguide case, an interference pattern due to surface and bottom bounces is formed that results in a breakdown of the range-depth pattern, with minimal impact on the radiation pattern versus bistatic angle. The consistency in the location of radiation pattern lobes across range and depth is illustrated in Fig. 4, 

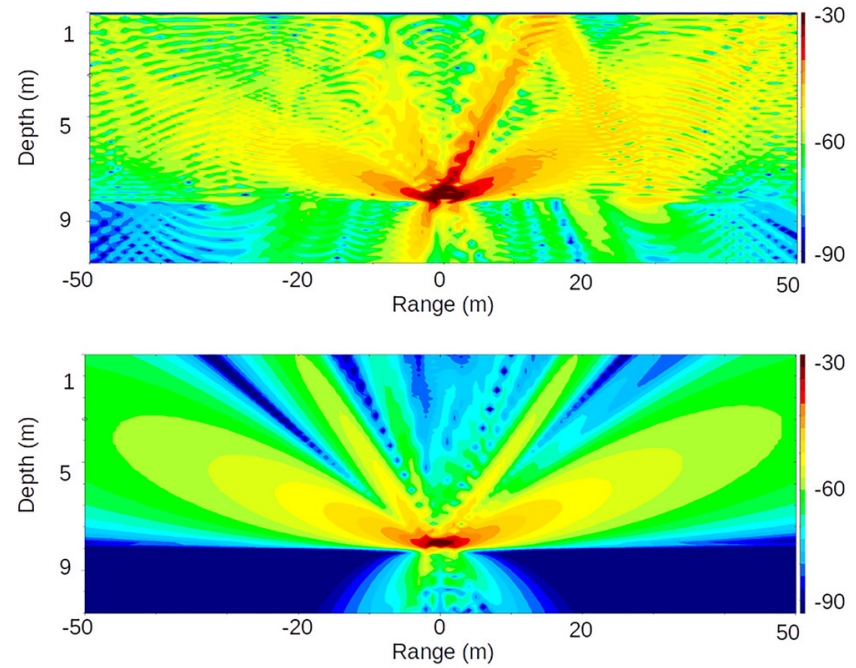

FIG. 3. (Color online) Bistatic scattering fields in range-depth space for a 0 deg aspect rigid cylinder, in a waveguide (above) versus in a halfspace (below). Color axis is simulated scattering field in $\mathrm{dB}$ assuming an incident plane wave at $0 \mathrm{~dB}$.

which shows the bistatic scattered field for a rigid 3-1 cylindrical target ( $1.5 \mathrm{~m}$ long, $0.5 \mathrm{~m}$ diameter) at three difference receiver depths and three aspect angles, with simulation parameters of a $15 \mathrm{~m}$ deep waveguide with a fluid sand bottom, a source frequency of $8000 \mathrm{~Hz}$, a source range of $100 \mathrm{~m}$ and source depth of $3 \mathrm{~m}$. The major features in these plots are radial in nature and persistent across depth. The interference pattern is observed in range and depth, but is less significant than the scattering amplitude changes with bistatic and aspect angle.

Figure 5 shows the types of scattering amplitude features that arise in the bistatic angle versus aspect angle space for four targets: an elastic air-filled sphere, a fluid-filled cylinder, and rigid cylinder. These plots are based on simulation results in a $15 \mathrm{~m}$ deep waveguide with a fluid sand bottom, an $8 \mathrm{kHz}$ source at $3 \mathrm{~m}$ depth and $100 \mathrm{~m}$ range, showing median scattering amplitudes between sampling ranges of $30-40 \mathrm{~m}$ and sampling depths of $3-5 \mathrm{~m}$.

This entire space is theoretically accessible to AUVs, but sensing scattering amplitude at the full range of bistatic and aspect angles is impractical. The goal of this multistatic scattering-based target characterization methodology is rapid fully autonomous target classification. It was therefore critical to identify which parts of this large space have the greatest utility for classification, so that AUVs can be deployed in data collection behaviors to efficiently estimate target parameters.

\section{B. Data collection behaviors}

To sense the multistatic scattered field with autonomous vehicles, an acoustic source would be mounted on a source vehicle. One or more AUVs fitted with hydrophone nose arrays, time synchronized data acquisition systems and acoustic processing payloads then act as receivers. A $1 \mathrm{~Hz}$ repeat rate is used because source firing and receiver recording are triggered underwater using synchronization of a chip scale atomic clock to global positioning system (GPS) pulse-per-second (PPS). ${ }^{27}$ Each second, the acoustic source fires, insonifying the target. Array data on each receiver is first processed in real time to track and localize target contacts. Once a target has been localized using a processing chain that includes beamforming, filtering, and tracking, a target classification mode is initialized for the estimated target location. The receiver vehicle that has localized a target would communicate via acoustic modem the target location to a source vehicle and additional receiver vehicles, instantiating target classification behaviors. A description of several possible multistatic sampling behaviors can be found in Ref. 28 .

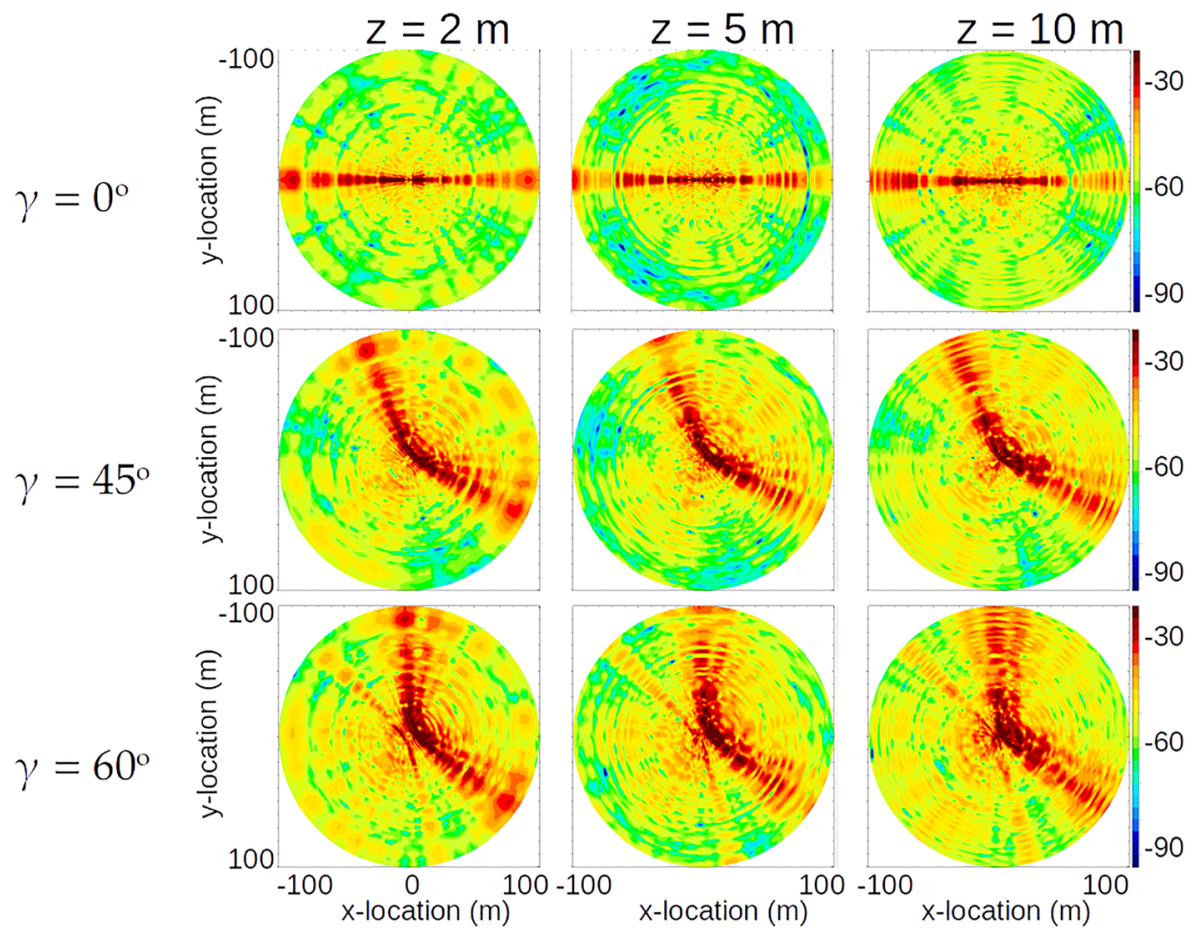

FIG. 4. (Color online) Bistatic scattering fields in range-range space at three aspect angles $\gamma$ and three depths $z$. Color axis on all plots is simulated scattering field in $\mathrm{dB}$ assuming an incident plane wave at $0 \mathrm{~dB}$. (a) Air-filled elastic sphere. (b) Fluid-filled cylinder. (c) Rigid cylinder. 


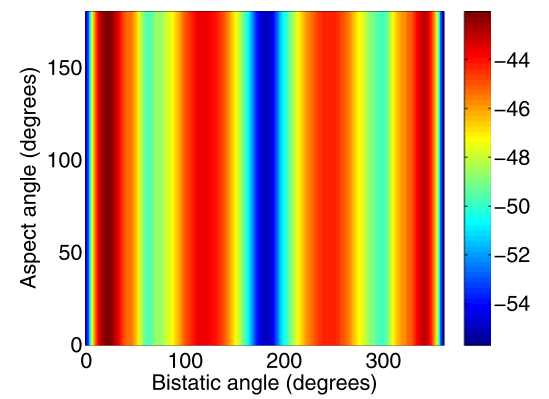

(a)

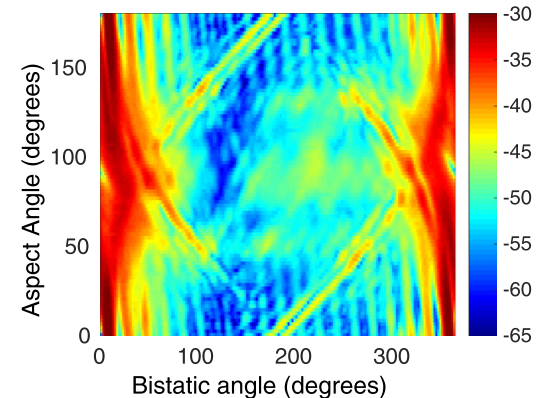

(b)

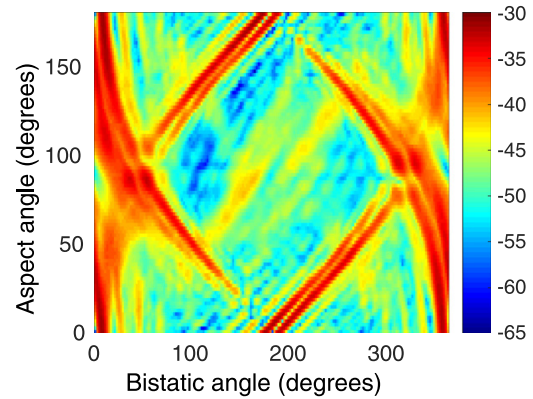

(c)

FIG. 5. (Color online) Comparison of bistatic/aspect angle dependence of scattering from sphere and cylinder targets, $8 \mathrm{kHz}$ source at $3 \mathrm{~m} \mathrm{depth}$ and $100 \mathrm{~m}$ range. Color axis on all plots is simulated scattering field in $\mathrm{dB}$ assuming an incident plane wave at $0 \mathrm{~dB}$.

In one promising multistatic sampling behavior, the constant-bistatic-angle or CBA behavior, the source vehicle and one or more receiver vehicles circle the estimated target location at constant radii while maintaining selected bistatic angles between source and receivers. By sampling the full range of aspect angles for a given bistatic angle, this behavior addresses an issue introduced by the absolute target orientation to the multistatic scattering problem. The receiving AUV does not know the absolute orientation of the target, so it is not actually able to measure target scattering amplitude versus aspect and bistatic angle, $A(\gamma, \theta)$. Instead, it measures target scattering amplitude versus source and bistatic angles, $A(\beta, \theta)$, where $\beta$ is the source angle (Fig. 6). Note that a bistatic angle of $0 \mathrm{deg}$ is monostatic (source and receiver colocated), a aspect angle of 0 deg has the target end-on to the source, and an aspect angle of $90 \mathrm{deg}$ results in a the target broadside to the source. A source angle of 0 occurs when the source is directly north of the target.

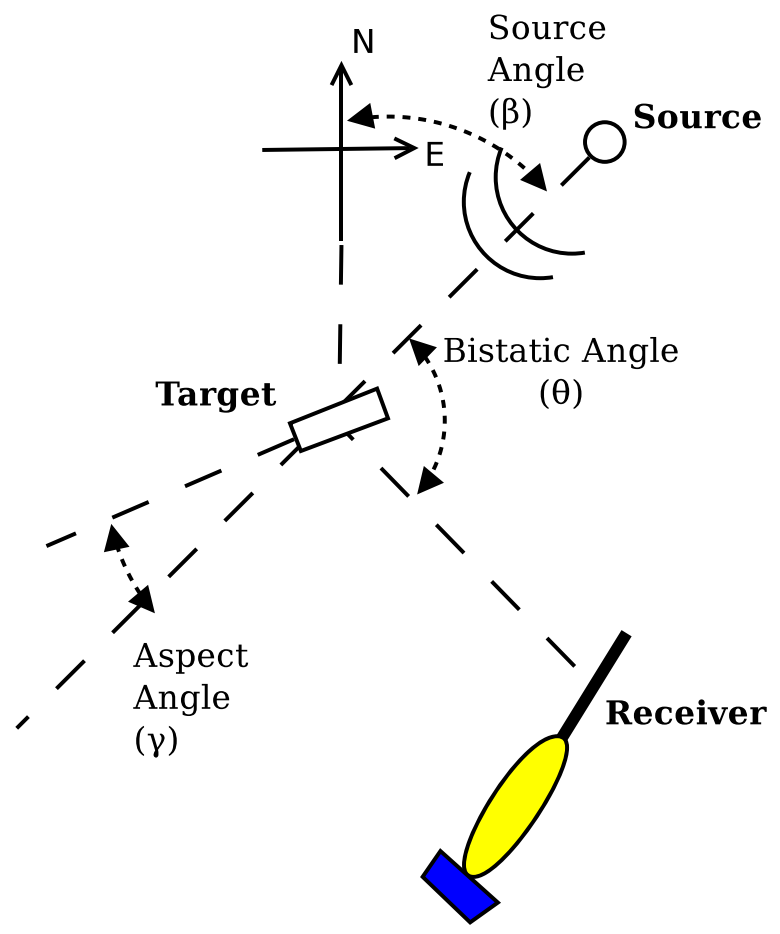

FIG. 6. (Color online) Bistatic angle $\theta$, aspect angle $\gamma$, and source angle $\beta$ in the horizontal plane.
Figure 7 illustrates this problem. When the target orientation changes, the map of scattering amplitude versus bistatic angle and receiving angle has an offset while containing the same information. This reality precludes using direct $A(\beta, \theta)$ for target discrimination, and it is not possible to directly sense $A(\gamma, \theta)$ without first measuring $\gamma-\beta$. While it is possible to use regression based on the target's scattered field to estimate $\gamma$, it requires a large amount of data ( $>15$ min at one sample per second), making an approach requiring $\gamma$ estimation impractical for rapid target classification. ${ }^{5}$ One sample is collected per second using our method because time synchronization is achieved by using a PPS signal to trigger both source firing and data recording.

By sampling all aspect angles at a single bistatic angle, however, a frequency analysis can be used to eliminate the offset. A Fourier transform is taken of $A(\beta)$ for a constant $\theta$, resulting in frequency components that can be used for target discrimination. These frequency components, such as those shown in Fig. 8, are the same regardless of the value of $\gamma-\beta$ and may be used to form classification models.

\section{Simulations}

To develop target characterization methods using multistatic data collection by vehicles in CBA behaviors, acoustic scattering fields were simulated for a variety of target types and shapes for several of environments. The SCATTOASES acoustic simulation package was used for all scattering simulations. This acoustic simulator produces three dimensional scattering fields from selected target and source parameters in a range-independent layered environment. ${ }^{9,26}$ An $8 \mathrm{kHz}$ source with $3 \mathrm{~m}$ depth and $50 \mathrm{~m}$ range was used
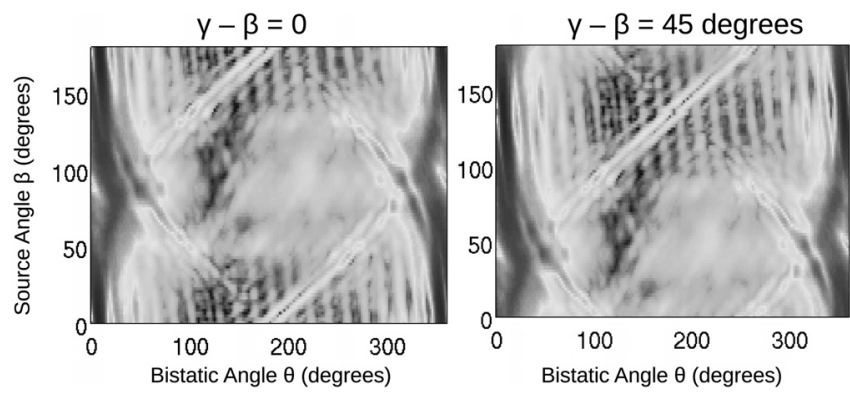

FIG. 7. Visualization of orientation impact on scattering amplitude versus bistatic and source angle. The targets are identical expect for a $45 \mathrm{deg}$ offset in target orientation. 


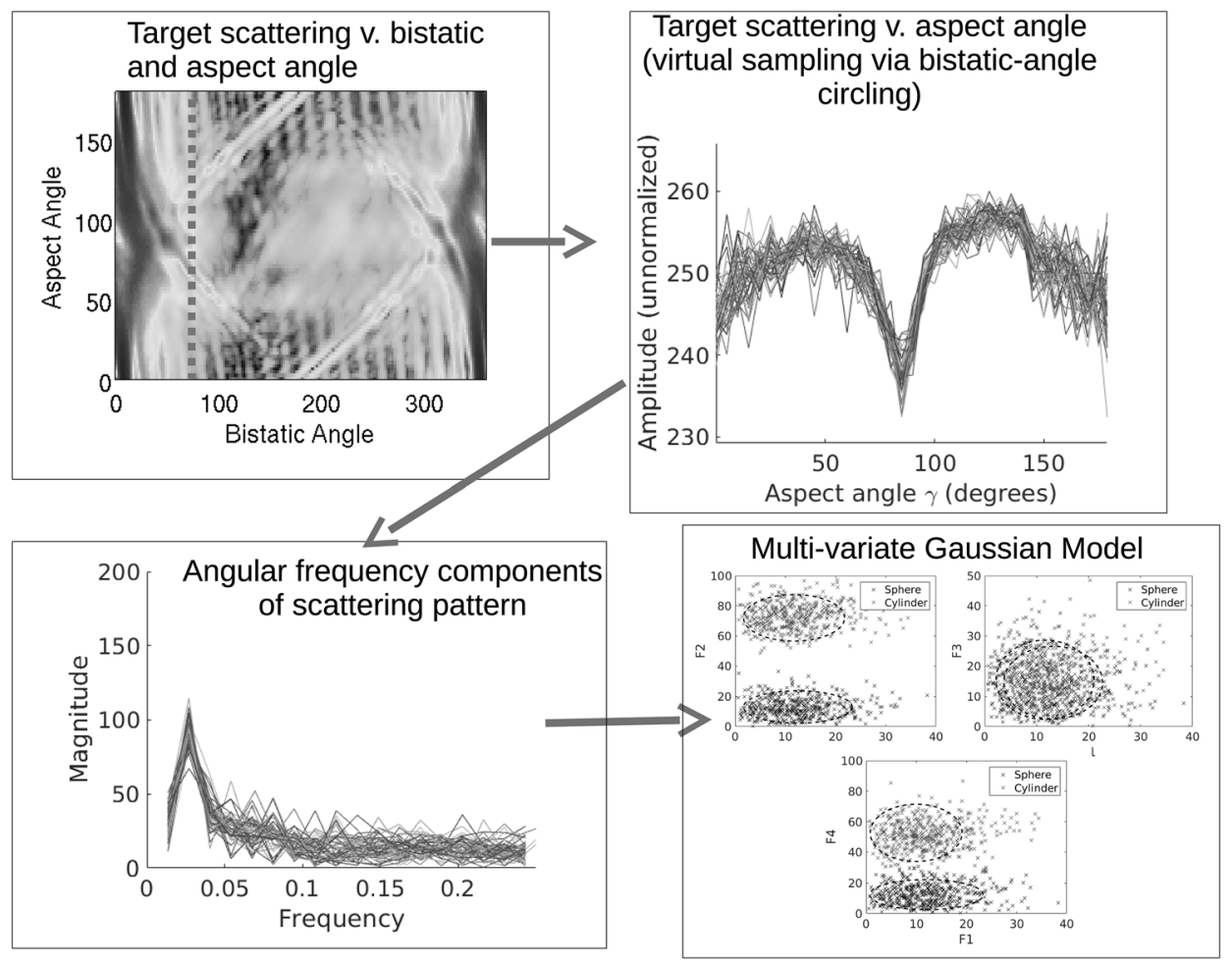

FIG. 8. Process for getting frequency components from multistatic scattering amplitudes: data is sampled along a constant bistatic angle then the Fourier transform is taken. The resulting frequency components provide features that are robust to changes in target orientation offset $\gamma-\beta$.

for all targets and environments. Scattered fields were calculated for bistatic and aspect angles in $2 \mathrm{deg}$ increments for each target/environment combination. Targets included spheres and cylinders of varying geometry and composition. Four environments were tested: $7 \mathrm{~m}$ deep with mud-oversand bottom, $7 \mathrm{~m}$ deep with sand bottom, $15 \mathrm{~m}$ deep with mud-over-sand bottom, and $15 \mathrm{~m}$ deep with a sand bottom. The scattered field for the rigid cylinder in the four environments is shown in Fig. 9.

Virtual sampling of each simulated scattered field was used to emulate data collection by receiver AUVs in CBA behaviors with a source vehicle. This virtual sampling involved psuedo-randomly selecting $N$ data points from the data at a particular bistatic angle, approximating data collected by vehicles attempting to circle the target at constant bistatic angle for $N$ seconds. First, a pseudo-random receiver range and depth is selected. Within the data with that range and depth, $N$ data points were chosen such that $\theta=\theta_{d}+\delta$, where $\theta_{d}$ is the desired constant bistatic angle, $\theta$ is the bistatic angle for a particular sample, and $\delta$ is an error in bistatic angle between vehicles. $\theta$ was not set equal to $\theta_{d}$ because, in the real world, bistatic angle will not be perfectly maintained between vehicles. $\delta$ was randomly assigned for each sample based on a Gaussian distribution $N(\mu, \sigma)$ with a mean $\mu=0$ and a standard deviation of $\sigma=5^{\circ}$. (An error of $\sigma=5^{\circ}$ is equivalent to a $2.6 \mathrm{~m}$ error at $30 \mathrm{~m}$ radius, a $4.4 \mathrm{~m}$ error at $50 \mathrm{~m}$ radius, and a $8.8 \mathrm{~m}$ error at $100 \mathrm{~m}$ radius.)

This sampling process is shown in Fig. 8. Each line in the amplitude and frequency component plots represents data for a single example.

\section{Target characterization}

The frequency components make convenient features for target discrimination in several ways. The data in this format is well-represented by a multivariate Gaussian, and the first five components were found to contain most of the relevant information for target discrimination.

At a given bistatic angle, each target can be modelled as a multivariate Gaussian distribution based on an set of

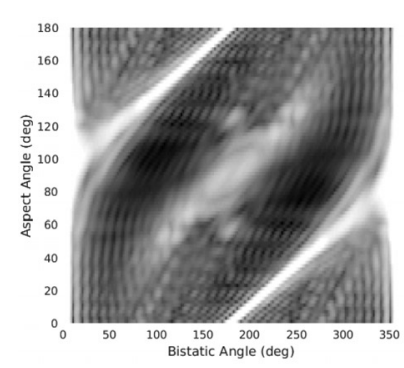

$15 \mathrm{~m}$, sand

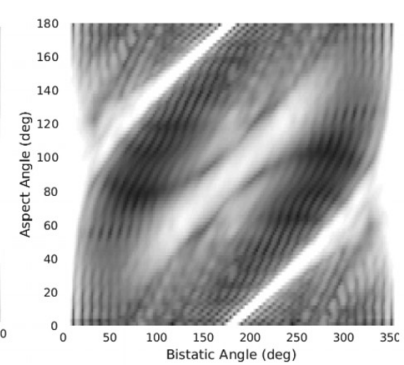

$7 \mathrm{~m}$, sand

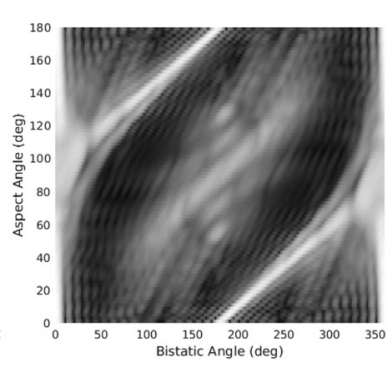

$15 \mathrm{~m}$, mud-over-sand

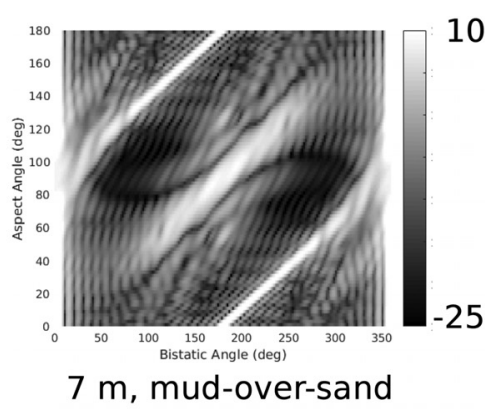

$7 \mathrm{~m}$, mud-over-sand

FIG. 9. Difference in aspect versus bistatic scattering field for the four tested environments for a rigid cylinder, $8 \mathrm{kHz}$ source at $50 \mathrm{~m}$ range. Scale scattering field in $\mathrm{dB}$. 
example paths generated using the virtual sampling methodology described above. New data at the same bistatic angle can be assessed compared to existing models using loglikelihood.

Each target $m$ is modelled as multivariate Gaussian distribution $N_{m}\left(\mu_{\mathbf{m}}, \Sigma_{m}\right)$, where $\mu_{\mathbf{m}}$ is a $K \times 1$ vector of means and $\Sigma_{m}$ is a $K \times K$ covariance matrix based on a data matrix $M_{m} . M_{m}$ is a $K \times N$ matrix, where $K$ is the number of frequency components used as features and $N$ is the number of training examples. The mean vector $\mu_{\mathbf{m}}$ is established by calculating the mean of $M_{m}$ for each feature and example, and $\Sigma_{m}$ is calculated by getting the covariance matrix. The final part of Fig. 8 shows the multivariate Gaussian for CBA sampling of a cylinder target in several 2D representations: the first frequency component versus the second, the third, and the fourth. In this example, the Gaussian model is shown to clearly capture the variability in the simulated data.

\section{E. Metrics for Gaussian modeling}

Gaussian information metrics such as Kullback-Leibler divergence $^{29}$ can be used to compare different data sets based on the Gaussian representations. If each target $m$ is represented using a multivariate Gaussian $N_{m}\left(\mu_{\mathbf{m}}, \Sigma_{m}\right)$, and the difference between the two targets (let us call them $m=1$ and $m=2$ ) can be represented using the Kullback-Leibler divergence:

$$
\begin{aligned}
D_{\mathrm{KL}}\left(N_{1}, N_{2}\right)= & \frac{1}{2} \operatorname{Tr}\left\{\Sigma_{2}^{-1} \Sigma_{1}+\left(\mu_{2}-\mu_{1}\right)^{T}\right. \\
& \left.\times \Sigma_{2}^{-1}\left(\mu_{2}-\mu_{1}\right)-K+\ln \left(\frac{\operatorname{det}\left(\Sigma_{2}\right)}{\operatorname{det}\left(\Sigma_{1}\right)}\right)\right\},
\end{aligned}
$$

where the index, e.g., the 1 in $\Sigma_{1}$, indicates the distribution number. New data can be compared to existing models $N_{m}$ using the log-likelihood probability that a new data vector $x$ with dimensions $K \times 1$ belongs to a multivariate Gaussian $N_{m}$ :

$$
L L_{m}(x)=-\frac{1}{2} \ln \left(\operatorname{det}\left(\Sigma_{m}\right)\right)-\frac{1}{2}(x-\mu)^{T} \Sigma_{m}^{-1}(x-\mu)-\frac{K}{2} 2 \pi,
$$

where $M_{m}$, and $\Sigma_{m}$ are mean and covariances of the Gaussian model.

Both $D_{\mathrm{KL}}$ and $L L$ may be used to assess the similarity of models at particular bistatic angles, e.g., how similar the CBA data is for a fluid-filled versus rigid cylinder at a bistatic angle of $90 \mathrm{deg}$. Log-likelihood is used to assign characteristics to new data: $L L_{m}(x)$ is calculated for each target model $m$. The likelihood that a given example $x$ belongs to the same class as model $m y_{m}$ can then be calculated as

$$
l\left(y(x)=y_{m}\right)=y_{m} \frac{e^{L L_{m}(x)}}{\sum_{m} e^{L L_{m}(x)}},
$$

where $\sum_{m} e^{L L_{m}(x)}$ is the sum of the likelihoods that the example belongs to all of the model values $y_{m}$, so that the probabilities always sum to zero.

These likelihoods can be used for classification or regression. In classification, the highest likelihood category is selected as the class:

$$
y(x)=\operatorname{argmax}_{y_{m}} y_{m}\left(y(x)=y_{m}\right) .
$$

In regression, likelihood is used as weights to estimate the characteristic property of example $x, y(x)$ :

$$
y(x)=\sum_{m} y_{m} l\left(y(x)=y_{m}\right) .
$$

This method of characterization has advantages of giving good similarity measurements across a wide range of candidate target types, providing good physical intuition of similarities between targets, and making confidence in an estimate easy to calculate. Accuracy can calculated as the percent of correct test classifications divided by the total classifications, and confidence is the likelihood that the example belongs to the selected class. The error for a given example $x, \epsilon$, is calculated as

$$
\epsilon(x)=y(x)-y_{\text {true }},
$$

where $y_{\text {true }}$ is the true value of $y$ and $y(x)$ is the regression estimate from Eq. (5). Accuracy of regression estimates are assessed based on the percentage of examples with less than $\alpha$ percent error compared to the maximum of the parameter being estimated, $P(|\epsilon|>\alpha)$.

\section{RESULTS}

The goal of this work was to investigate the use of multistatic scattering amplitude features for target discrimination. Using simulation and virtual sampling, we were able to look at and compare data that an AUV might acquire at different bistatic angles to a source vehicle for multiple targets and environments. The following analysis examines the impact of target geometry, target composition and environment on multistatic data. We identify critical features in the bistatic angle-aspect angle sampling space accessible to AUVs in constant-bistatic-angle behaviors, and show target classification and parameter estimation. The analysis methods described here could be applied to a number of different target parameter estimation or classification problemsthese are some sample problems based on things easily tested in the existing simulation environment.

An analysis of bistatic angle utility for target discrimination is presented along with modeling and performance with selected single bistatic angles. Kullback-Leibler divergence $\left(D_{\mathrm{KL}}\right)$ was used to assess the bistatic angles for which Gaussian models provide the maximum separation between target types. The performance of classification based on loglikelihood was assessed for the best bistatic angle using on an independently drawn simulation data set. Unless otherwise indicated, the $15 \mathrm{~m}$ deep waveguide with a sand bottom is used in simulations.

Simulation results showed that different bistatic angles reveal information on different target parameters, and that some bistatic angles demonstrate better robustness to changes in environment. This aligns with Blondel's multistatic classification results, ${ }^{13}$ which showed that different bistatic angles provided different information on target characteristics over the three aspect angles he examined. 
All simulation results presented here are based on training sets composed of 1000 training examples per class in the trained model, with each example consisting of $N=300$ data points from the simulated scattered field, selected as described in Sec. II C. All classification or regression results are based on independently drawn test sets composed of 1000 test examples per class, with each example consisting of $N=300$ data points from the simulated scattered field, selected as described in Sec. II C. All targets were simulated as proud on the top layer of sediment.

\section{A. Feature space}

The first six frequency components were used as features throughout. This number of components was chosen to limit computational complexity while maximizing separation between classes in the sphere versus cylinder case as indicated by $D_{\mathrm{KL}}$ : increasing from six to seven features does not change information difference between sphere and cylinder target significantly.

\section{B. Sphere versus cylinder}

The baseline classification problem tested using this method was sphere versus cylinder classification. The sphere class is set to 1 and the cylinder class to -1 . The model was trained on examples from the sphere and the rigid cylinder examples, and sphere, rigid cylinder, fluid cylinder, and airfilled cylinder examples were classified.

\section{Bistatic angle selection}

Kullback-Liebler divergence between sphere and cylinder Gaussian models for multiple cylinder compositions is shown in Fig. 10. This plot shows maximum likelihood of correct classification should occur at $150 \mathrm{deg}$ and minimum likelihood of correct classification should occur at $45 \mathrm{deg}$, though most bistatic angles provide reasonable separation for sphere versus cylinder over all cylinder types. Kullback-Leibler results on the training set were used to evaluate the "best" bistatic angle as bistatic angle selection is considered part of the training process.

\section{Classification}

Classification accuracy was calculated for 45 and $150 \mathrm{deg}$ (best and worst bistatic angles) based on the application of Eq. (4) to 1000 random test examples. Class for a particular example is determined as the class with maximum likelihood, and accuracy is calculated based on the number of true positives and negatives divided by the total number of test examples. Both bistatic angles resulted in excellent separation and near-perfect classification. A bistatic angle of $45 \mathrm{deg}$ resulted in $99.8 \%$ accuracy and a bistatic angle of $150 \mathrm{deg}$ resulted in a $100 \%$ accuracy across all 4000 test examples (sphere, rigid cylinder, fluid-filled cylinder, airfilled cylinder). A bistatic angle of $0 \mathrm{deg}$ (monostatic case) yielded a $98.7 \%$ accuracy, similar performance to the best multistatic angle.

While near-perfect accuracy might be generally suspect using a machine learning approach, we feel that it is not an artifact of our training process in this case because training and test sets are independently and randomly selected, half of the test examples were drawn from targets not included in the initial model, because of the clear difference between the signals, and because our prior results showed similar accuracies on actual AUV data using a different approach. For a constant bistatic angle, the sphere has constant scattering amplitude. Noise is added on top of that with navigation error, but even with that error the frequency components are all close to zero in our feature space. The cylinder, on the other hand, has features with constant bistatic angle that cause strong variations in the frequency components. This difference is the basis of the very high classification accuracy. Furthermore, $100 \%$ accuracies were observed with similar numbers of samples in the bistatic classification case where experimental AUV-collected bistatic scattering data were classified based on simulation-derived models. For these reasons we have some confidence that the reported $100 \%$ accuracy is not due to over-training.

\section{Target composition}

In the target characterization process, one parameter of interest is target composition, i.e., what the target is filled

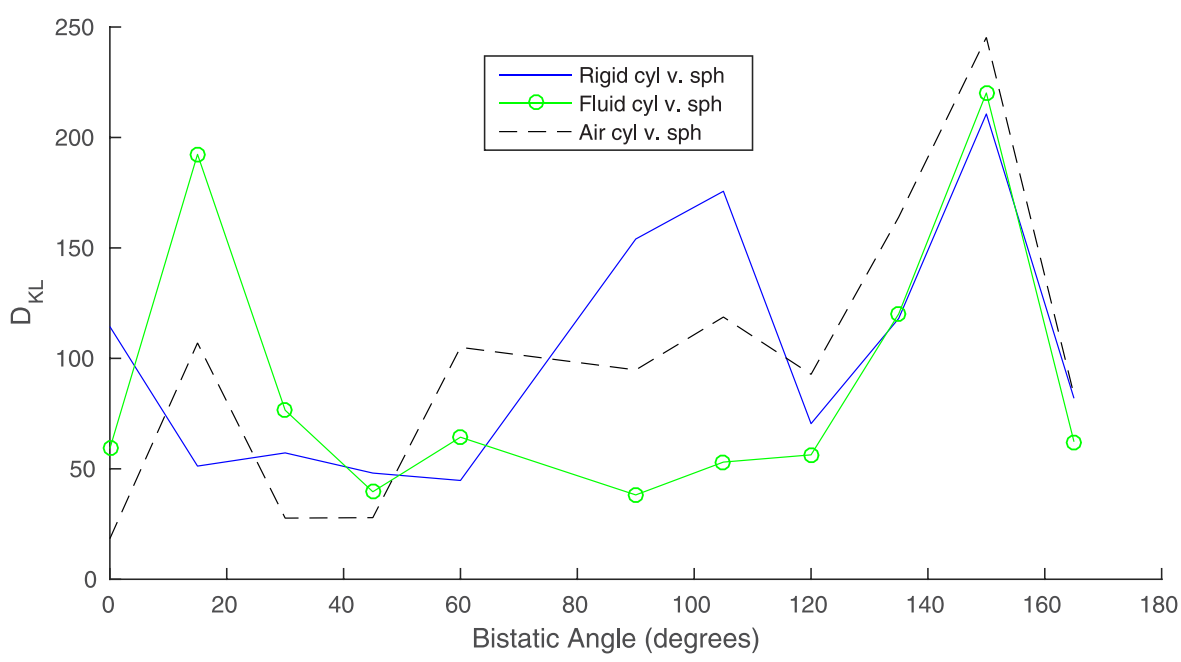

FIG. 10. (Color online) KullbackLeibler divergence versus bistatic angle for cylinder versus sphere classification. For all three cylinder types, a bistatic angle of $150 \mathrm{deg}$ results in the largest information difference between sphere and cylinder models. $45 \mathrm{deg}$ results in the lowest information difference between the models. 


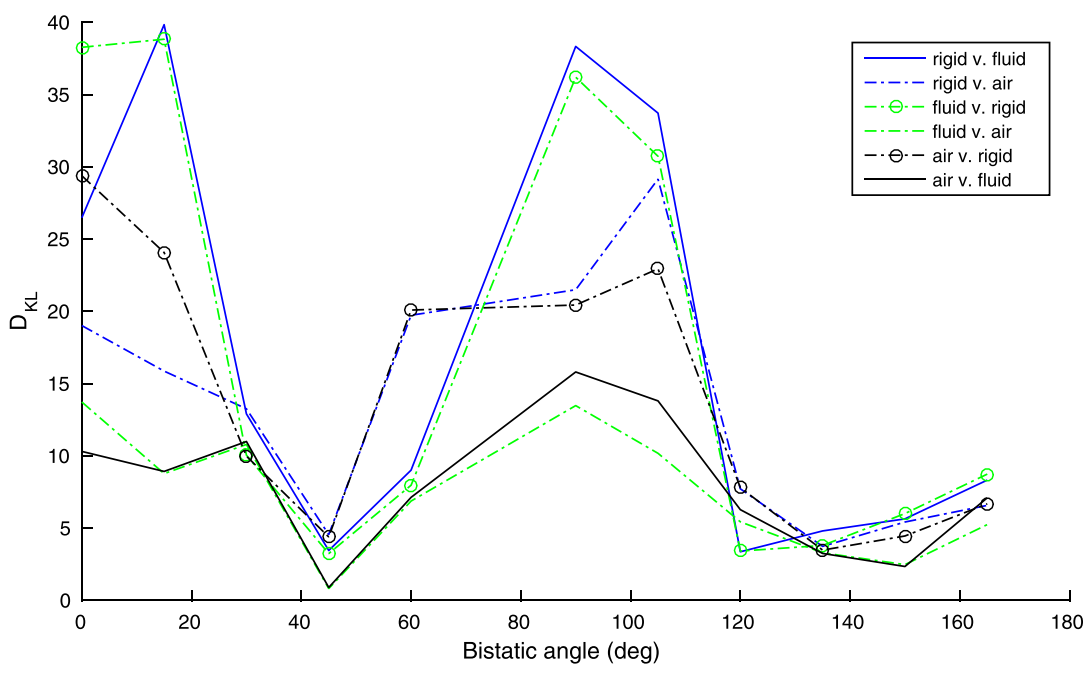

FIG. 11. (Color online) KullbackLeibler divergence versus bistatic angle for cylinders with different compositions classification. For all three cylinder types, a bistatic angle of $90 \mathrm{deg}$ results in the largest information difference between models. Forty five degrees results in the lowest information difference between the models.

with. This is important in distinguishing, for example, clutter from unexploded ordinance in the munition detection, classification, and mitigation mission. The bistatic angle that gave the greatest likelihood for correct classification based on training data were determined to be $90 \mathrm{deg}$, and the worst bistatic angle was again $45 \mathrm{deg}$, as shown in Fig. 11. One thousand independently drawn test examples were classified using Eq. (4). Overall classification accuracy was found to be $99.8 \%$ at $90 \mathrm{deg}, 82.5 \%$ at $45 \mathrm{deg}$ and $99.2 \%$ at $0 \mathrm{deg}$. Multistatic at a bistatic angle of $90 \mathrm{deg}$ therefore provides little advantage over monostatic.

\section{Target volume and aspect ratio}

Another parameter of interest is target volume. Cylindrical targets with the following dimensions were simulated:

Targets 1 through 4 were used for training models, and regression was run to estimate the volume of independent examples from targets 5 and 6 . A greater number of training and test targets would improve performance, but the computational time is significant to simulate the full set of bistatic and aspect angles for each target, so this training set of 4 target models and test set of 2 models was used. Two hundred fifty five degrees was found to be the bistatic angle with best

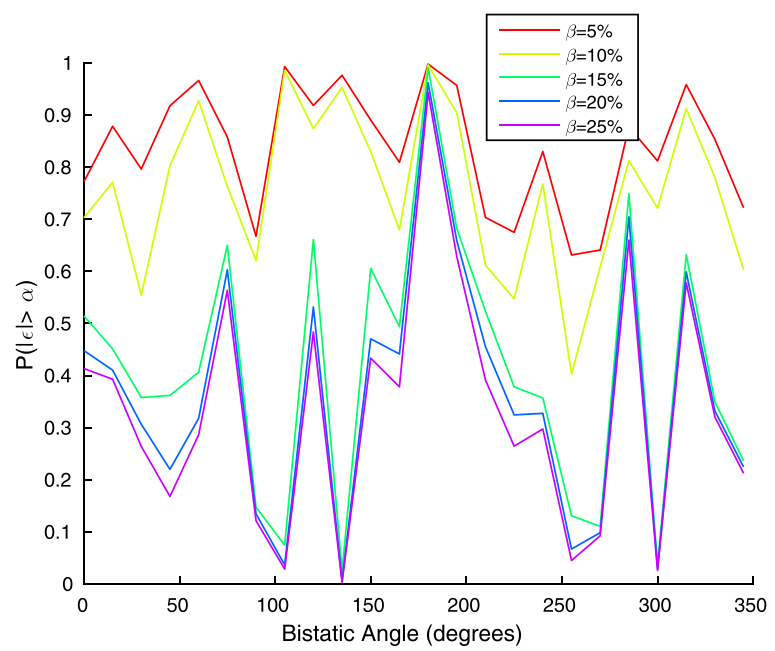

FIG. 12. (Color online) Combined volume accuracy for targets 5 and 6 versus bistatic angle for different values of $\alpha$. target volume discrimination. Figure 12 shows the probability of an error greater than $\alpha$ for targets 5 and 6 across all bistatic angles. This shows the worst performance at 180 degrees and the best at 255 degrees: volume estimates of $y(x)$ at a bistatic angle of 255 degrees is shown in Fig. 13. This shows the worst performance at $180 \mathrm{deg}$, and the best performance at $255 \mathrm{deg}$. The monostatic case performs poorly, with a $50 \%$ chance of errors of greater than $15 \%$ of maximum volume in the training set. In comparison, the $255^{\circ}$ case had a $10 \%$ chance of errors greater than $15 \%$ of maximum volume.

The regression is not highly accurate, but at least it does indicate which target is larger.

Interestingly, a different bistatic angle may be selected to estimate target aspect ratio (height divided by diameter): the aspect ratio of targets 5 and 6 are both 3 . The performance versus bistatic angle is shown in Fig. 14. The "good" bistatic angles are significantly different than for volume estimation: in fact, $255 \mathrm{deg}$ is among the worst choices for estimating geometric aspect ratio. Instead, 105 and $165 \mathrm{deg}$ stand out as particularly good. Again, a bistatic angle of 0 deg gives poor performance.

As with volume estimation, the aspect ratio regression results using this technique are not precise, but they do give a general idea of target aspect ratio.

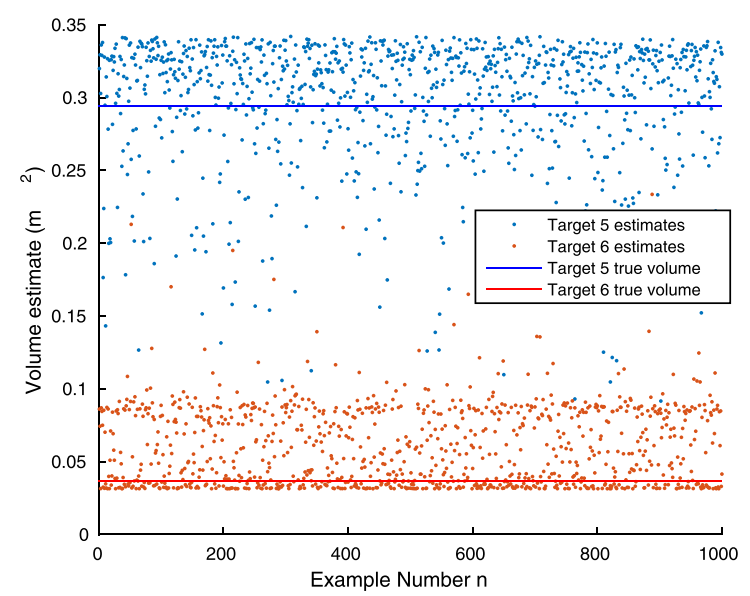

FIG. 13. (Color online) Volume estimation for targets 5 and 6 with a bistatic angle of $255 \mathrm{deg}$. 


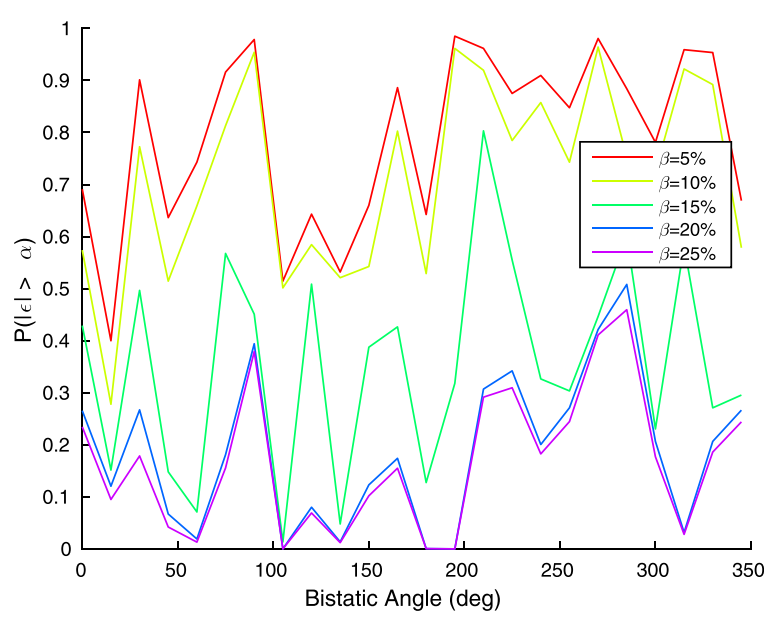

FIG. 14. (Color online) Combined aspect ratio accuracy for targets 5 and 6 versus bistatic angle for different values of $\alpha$.

This technique clearly provides some information on target volume and aspect ratio, but the estimates are coarse. More accurate estimates would likely result from using multifrequency data and/or applying a different regression technique using a similar feature space. The most interesting part of this result is that different bistatic angles yield target size versus aspect information and that the monostatic result is poor: these facts could be exploited in multibistatic angle behaviors or classification schemes.

\section{E. Robustness to environment}

All of the above classifications and regressions used a constant, known environment for classification or regression. An important quality of any target characterization technique, however, is robustness to changes in environmental characteristics such as bottom type and water depth.

We tested the impact of environment on the Gaussian models by first simulating cylinder 1 from Table $\mathrm{I}$ in four environments:

A model was trained based on 1000 training examples for each environment, shown in Table II, and the Kullback-Liebler divergence was then calculated between the model for each environment and all of the others at the tested bistatic angles. The Kullback-Liebler divergence gives a sense of how much and at what bistatic angles the change of environment affects the model, as shown in Fig. 15.

The maximum information difference based on environmental change occurs at a bistatic angle of $45 \mathrm{deg}$. The

TABLE I. Cylinder target geometries used in volume and aspect estimation.

\begin{tabular}{lcccc}
\hline \hline Number & Radius $(\mathrm{m})$ & Height $(\mathrm{m})$ & Volume $\left(\mathrm{m}^{2}\right)$ & $\begin{array}{c}\text { Aspect ratio } \\
\text { (height/diameter) }\end{array}$ \\
\hline 1 & 0.33 & 1.5 & 0.342 & 2.27 \\
2 & 0.25 & 1 & 0.1962 & 2 \\
3 & 0.1667 & 1 & 0.0872 & 3 \\
4 & 0.1 & 1 & 0.0314 & 5 \\
5 & 0.25 & 1.5 & 0.2945 & 3 \\
6 & 0.125 & 0.75 & 0.0368 & 3 \\
\hline \hline
\end{tabular}

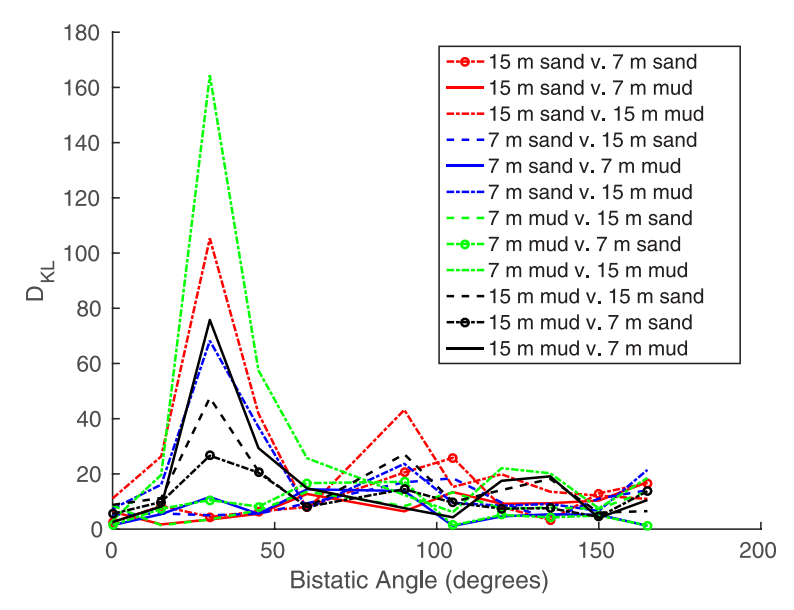

FIG. 15. (Color online) Kullback-Liebler divergence for cylinder 1 in the environments lists in Table II. The maximum information difference occurs at $45 \mathrm{deg}$.

environment will have the greatest affect on target classification or regression at this angle, with lesser effects at other angles. Significantly, this angle was observed to have the worst performance for classification of targets based on shape or composition.

\section{CONCLUSIONS AND SUGGESTIONS FOR FUTURE WORK}

The preliminary analysis is promising: the models result in good classification separation between targets with different compositions and shapes. Regression results for estimating target volume and aspect angle are promising, but will require further investigation to improve overall accuracy, perhaps by incorporating multiple frequencies. Overall performance is highly dependent on bistatic angle, with different bistatic angles providing information about different target characteristics or the environment. Zero degree bistatic angle (monostatic case) did not give maximum information difference for any of the target characteristics we examined, though it provided nearly identical performance to the best multistatic angle for both sphere/cylinder classification and target composition classification. Data from multiple bistatic angles could be combined into even more effective classifiers.

Further investigation is needed to understand the frequency dependence of this phenomena, its application to different types of targets, and its translation into the field using AUVs for data collection. Discrimination of symmetric targets also needs to be addressed, perhaps by tweaking the feature space to include some constant-aspect data.

The information garnered in simulation on the importance of bistatic angle on classification of different features

TABLE II. Compared environments in robustness to environment evaluation.

\begin{tabular}{lcc}
\hline \hline Env. number & Water depth $(\mathrm{m})$ & Bottom type \\
\hline 1 & 15 & Sand \\
2 & 7 & Sand \\
3 & 7 & Mud \\
4 & 15 & Mud \\
\hline \hline
\end{tabular}


has important implications in designing vehicle behaviors in actual experiments. The success in simulation, coupled with the BayEx'14 experiment results, suggests that it is plausible to use mobile source and receiver vehicles sensing acoustic scattering amplitude to discriminate targets. Experiments should be conducted to compare multistatic data in simulation versus experiments, and to demonstrate this classification methodology on vehicles that the technique is designed for: low-cost AUVs like the General Dynamics Bluefin SandShark.

\section{ACKNOWLEDGMENTS}

The authors would like to thank the Laboratory for Autonomous Marine Sensing Systems group at MIT. This work was supported by Battelle.

${ }^{1}$ M. Zampolli, F. B. Jensen, and A. Tesei, "Review of target strength information for waterside security application," in Proc. WSS 2008, Technical University of Denmark, 25-28 Aug. 2008.

${ }^{2}$ P. Blondel and A. Caiti, Eds., Buried Waste in the Seabed-Acoustic Imaging and Bio-toxicity (Results from the European SITAR Project) (Springer-Praxis, Chichester, UK, 2007).

${ }^{3}$ P. Blondel, P. F. Dobbins, N. Jayasundere, and M. Cosci, "High-frequency bistatic scattering experiments using proud and buried targets," in Acoustic Sensing Techniques for the Shallow Water Environment (Springer, Netherlands, 2006), pp. 155-170.

${ }^{4}$ E. M. Fischell and H. Schmidt, "Classification of underwater targets from autonomous underwater vehicle sampled bistatic acoustic scattered fields," J. Acoust. Soc. Am. 138, 3773-3784 (2015).

${ }^{5}$ E. M. Fischell and H. Schmidt, "Supervised machine learning for estimation of target aspect angle from bistatic acoustic scattering," IEEE J. Ocean Eng. PP, 1-11 (2017).

${ }^{6} \mathrm{R}$. Linnehan and J. Schindler, "Multistatic scattering from moving targets in multipath environments," in 2009 IEEE Radar Conference, Pasadena, CA, 2009, pp. 1-6.

${ }^{7}$ E. Hanle, "Survey of bistatic and multistatic radar," IEE Proceedings F (Communications, Radar and Signal Processing) 133(7), 587-595 (1986).

${ }^{8}$ M. Cherniakov, R. S. A. R. Abdullah, P. Jančovič, M. Salous, and V. Chapursky, "Automatic ground target classification using forward scattering radar," IEE Proc.—Radar, Sonar Navigation 153(5), 427-437 (2006).

${ }^{9} \mathrm{H}$. Schmidt and J. Lee, "Physics of 3-D scattering from rippled seabeds and buried targets in shallow water," J. Acoust. Soc. Am. 105, 1605-1617 (1999).

${ }^{10}$ M. Zampolli, D. S. Burnett, F. B. Jensen, A. Tesei, H. Schmidt, and J. B. Blottman III, "A finite-element tool for scattering from localized inhomogeneities and submerged elastic structures," AIP Conf. Proc. 728(1), 464-471 (2004).

${ }^{11}$ H. Schmidt, I. Veljkovic, and M. Zampolli, "Bistatic scattering from buried targets in shallow water-experiment and modelling," in Proceedings of the 7th European Conference on Underwater Acoustics, Delft, The Netherlands, 2004.
${ }^{12}$ P. F. Dobbins, N. Jayasundere, and P. Blondel, "Multiple-aspect imaging of seafloor targets - analyses of tank experiment datasets," in Proc. 5th ECUA, Delft, The Netherlands (2004), pp. 469-474.

${ }^{13} \mathrm{P}$. Blondel, "Rapid distinction of dumpsite objects using Multiple-Aspect Scattering-Results from scaled tank experiments," J. Acoust. Soc. Am. 123, 3948 (2008).

${ }^{14}$ Z. J. Waters, H. J. Simpson, A. Sarkissian, S. Dey, B. H. Houston, J. A. Bucaro, and T. J. Yoder, "Bistatic, above-critical angle scattering measurements of fully buried unexploded ordnance (UXO) and clutter," J. Acoust. Soc. Am. 132(5), 3076-3085 (2012).

${ }^{15}$ L. Haumesser, A. Baillard, D. Decultot, and G. Maze, "Experimental identification of finite cylindrical shell vibration modes," J. Acoust. Soc. Am. 111, 2034-2039 (2002).

${ }^{16}$ J. A. Bucaro, H. Simpson, L. Kraus, L. R. Dragonette, T. Yoder, and B. H. Houston, "Bistatic scattering from submerged unexploded ordnance lying on a sediment," J. Acoust. Soc. Am. 126(5), 2315-2323 (2009).

${ }^{17}$ Y. H. Ji, G. H. Byun, J. S. Kim, H. S. Bae, and W. S. Kim, "Multi-static scattering characteristics of submerged objects with experimental investigation," J. Acoust. Soc. Am. 134(5), 4113 (2013).

${ }^{18}$ K. D. LePage and H. Schmidt, "Bistatic synthetic aperture imaging of proud and buried targets from an AUV," IEEE J. Ocean. Eng. 27(3), $471-483$ (2002).

${ }^{19}$ P. Blondel and N. G. Pace, "Bistatic sonars: Sea trials, laboratory experiments and future surveys," Arch. Acoust. 34, 95-109 (2009).

${ }^{20}$ K. L. Williams, S. G. Kargl, E. I. Thorsos, D. S. Burnett, J. L. Lopes, M. Zampolli, and P. L. Marston, "Acoustic scattering from a solid aluminum cylinder in contact with a sand sediment: Measurements, modeling, and interpretation," J. Acoust. Soc. Am. 127(2), 3356-3371 (2010).

${ }^{21}$ M. Zampolli, A. L. Espana, K. L. Williams, S. G. Kargl, E. I. Thorsos, J. L. Lopes, J. L. Kennedy, and P. L. Marston, "Low- to mid-frequency scattering from elastic objects on a sand sea floor," J. Comp. Acoust. 20(2), 1240007 (2012).

${ }^{22}$ J. A. Bucaro, B. H. Houston, M. Saniga, L. R. Dragonette, and T. Yoder, S. Dey, L. Kraus, and L. Carin,"Broadband acoustic scattering measurements of underwater unexploded ordnance (UXO)," J. Acoust. Soc. Am. 123, 738-746 (2008).

${ }^{23}$ S. Yildiz, P. Roux, S. T. Rakotonarivo, C. Marandet, and W. A. Kuperman, "Target localization through a data-based sensitivity kernel: A perturbation approach applied to a multistatic configuration." J. Acoust. Soc. Am. 135(4), 1800-1807 (2014).

${ }^{24}$ R. Lum, A. Balasuriya, and H. Schmidt, "Multistatic processing and tracking of underwater target using autonomous underwater vehicles," in Proc. 3rd Int. Conf. Underwater Acoustic Measurements: Technologies and Results, Nafplion, Greece (June 2009).

${ }^{25}$ A. Tesei, W. L. J. Fox, A. Maguer, and A. Lovik, "Target parameter estimation using resonance scattering analysis applied to air-filled, cylindrical shells in water," J. Acoust. Soc. Am. 108, 2891-2900 (2000).

${ }^{26}$ SCATT-OASES3D User's manual. Revision 2. http://lamss.mit.edu/ lamss/docs/scatt_manual.pdf (Last viewed September 13, 2017).

${ }^{27}$ E. Fischell, T. Schneider, and H. Schmidt, "Design, implementation and characterization of precision timing for bistatic acoustic data acquisition," IEEE J. Ocean. Eng. 41, 583-591 (2015).

${ }^{28}$ E. M. Fischell and H. Schmidt, "AUV behaviors for collection of bistatic and multistatic acoustic scattering data from seabed targets," in IEEE International Conference on Robotics and Automation (ICRA) (2016), pp. 2645-2650.

${ }^{29}$ S. Kullback and R. A. Leibler, "On information and sufficiency," Ann. Math. Stat. 22(1), 79-86 (1951). 